\title{
Effects of the electric field on the self-trapping excited states in conjugated polymers
}

\author{
Rou-li Fu \\ National Laboratory for Infrared Physics, Shanghai Institute of Technical Physics, \\ Chinese Academy of Sciences, Shanghai 200083, China \\ Guang-yu Guo \\ Department of Physics, National Taiwan University, Taiwan 10617, Taibei, China \\ Xin Sun \\ Department of Physics, Fudan University, Shanghai 200433, China
}

(Received 22 February 2000)

\begin{abstract}
We demonstrated that electric fields have strong impacts on the self-trapping excited states in conjugated polymers by numerically simulated calculations. Applied electric fields induce a displacement of positive and negative charges in a self-trapping excited state. Weak or moderate electric fields transfer part of the charges, so the self-trapping excited states in conjugated polymers are polarized. The degree of polarization increases linearly with the electric field $E$. The polarization of single-excited states is normal. It is worth to note that a self-trapping biexcited state shows negative polarization, i.e., the negative charges move along the direction of the electric field, while the positive charges drift in the opposite direction. Such behavior, which would be of interest both fundamentally and technologically, can be understood based on the quantum theory of polarization. Strong fields transfer all the charges in a self-trapping excited state, leading to the field dissociation of self-trapping excited states. The result is consistent qualitatively with the recent relevant experiments in conjugated polymers.
\end{abstract}

\section{INTRODUCTION}

Quite a lot of research ${ }^{1-6}$ has been focusing on the electroluminescence (EL) of conjugated polymers in recent years, due to possible applications of these materials as active components in light-emitting devices. ${ }^{7}$ As quasi-onedimensional (1D) systems, conjugated polymers have the characteristic of low-dimensional instability. ${ }^{8-10}$ The extra electron and hole are to induce the deformation of bond structure in low-dimensional systems, ${ }^{8-15}$ leading to the formation of the self-trapping excited states, e.g., bipolaron excitons $^{11-14}$ or nonlinear excitons, ${ }^{15}$ etc. Since the EL of conjugated polymers is thought to be related to these selftrapping excited states ${ }^{2,3}$ and the EL of conjugated polymers is due to the external field, it is thus essential to study the electric-field behavior of these states, i.e., how the selftrapping excited states in conjugated polymers behave in an applied electric field and if the behavior is the same as that of an electron-hole pair in conventional inorganic semiconductors in an applied field.

It is found that the applied electric fields has strong effects on the self-trapping excited states of conjugated polymers. Weak or moderate fields polarize the self-trapping excited states. The degree of polarization increases linearly with the electric field $E$. It is worth mentioning that a self-trapping biexcited state shows negative polarization, which would be of great interest both in basic principles and in technology. Strong fields split the self-trapping excited states directly into a hole polaron and an electron polaron, thus leading to photoluminescence quenching. The calculation results are compared with the recent relevant experiments. ${ }^{1-6}$

The presentation is organized as follows. An outline of the methods used in the calculations is given in Sec. II. Section III describes the formation of self-trapping excited states under electric fields, their behavior including phenomena in the presence of electric fields, and the possible origins of the phenomena. The significance and possible applications of the phenomenon, negative polarization, are discussed in Sec. IV. Section V contains a summary.

\section{METHODOLOGY}

For conjugated polymers, the basic physical process is determined by electron-lattice and electron-electron interactions. ${ }^{8}$ Regarding a conjugated polymer as a finite chain of $N \mathrm{CH}$ groups with $N$ electrons in the $\pi$ band, Stafstrom and $\mathrm{Chao}^{16}$ used the tight-binding model (SSH model ${ }^{17}$ ) including electron-lattice interactions to study the polymer in a degenerate ground state and under zero electric field and without considering the electron-electron interactions. On the basis of the Hamiltonian used in Ref. 16, we appended the symmetry-breaking term ${ }^{8}$ corresponding to nondegenerate ground states since conjugated polymers with EL have nondegenerate ground states. ${ }^{7}$ Thus, the electronlattice interactions $H_{e-p}$ can be written as

$$
\begin{aligned}
\hat{H}_{e-p}= & -\sum_{n, s}\left[t_{0}-\alpha\left(u_{n+1}-u_{n}\right)+(-1)^{n} t_{e}\right] \\
& \times\left(\hat{C}_{n+1, s}^{+} \hat{C}_{n, s}+\text { H.c. }\right),
\end{aligned}
$$

where $t_{e}$ is the symmetry-breaking term, determined by the energy difference between phase $A$ and phase $B$ of the nondegenerate ground states; $t_{0}$ is the electron hopping probabil- 
ity, and $\alpha$ the electron-lattice interacting constant. $C_{n, s}^{+}$and $C_{n, s}$ are the creation and annihilation operators of an electron on site $n$ with spin $s$, respectively.

The elastic energy of the system is

$$
H_{\text {elastic }}=\frac{1}{2} K \sum_{n}\left(u_{n+1}-u_{n}\right)^{2}+\sum_{n} K^{\prime}\left(u_{n+1}-u_{n}\right),
$$

where the linear term is introduced ${ }^{18}$ to remedy the unstability of the SSH Hamiltonian with respect to dimerization and uniform compression of all the bonds, which originates from the fact that the electron energy is linear with $u_{n}$ while the elastic energy is quadratic with $u_{n} .{ }^{16,18}$

The electron-electron interactions can be described by the extended Hubbard model, which will be treated in an unrestricted Hartree-Fock approximation ${ }^{19}$

$$
\begin{aligned}
H_{e-e}= & \frac{U}{2} \sum_{n}\left(C_{n, s}^{+} C_{n, s}-\frac{1}{2}\right)\left(C_{n,-s}^{+} C_{n,-s}-\frac{1}{2}\right) \\
& +V \sum_{n, s, s^{\prime}}\left(C_{n, s}^{+} C_{n, s}-\frac{1}{2}\right)\left(C_{n+1, s^{\prime}}^{+} C_{n+1, s^{\prime}}-\frac{1}{2}\right),
\end{aligned}
$$

where $U$ is the usual on-site Hubbard energy and $V$ represents the nearest neighbor Coulomb interaction, and $\frac{1}{2}$ in the bracket ensures the charge neutrality.

In the meanwhile, assuming that a uniform electric field $E$ is along the chain direction, the additional potential energy due to the field is then

$$
H_{E}=\sum_{n} e \operatorname{EnaC}_{n, s}^{+} C_{n, s} .
$$

Thus, the Hamiltonian for electrons in a conjugated polymer with nondegenerate ground states takes the form of

$$
\hat{H}_{e}\left(\left\{u_{n}\right\}\right)=\hat{H}_{e-p}+\hat{H}_{e-e}+\hat{H}_{E} .
$$

Let the order parameter $\phi_{n}=(-1)^{n} u_{n}$, so the variations of the bond length $u_{n+1}-u_{n}$ is then $(-1)^{n+1}\left(\phi_{n}+\phi_{n+1}\right)$. $\left\{\phi_{n}^{0}\right\}$ and $\delta \phi_{n}$ represent the static equilibrium configuration of the system and the small displacement from the equilibrium configuration, respectively. When $\delta \phi_{n} \ll \phi_{n}^{0}$, the energy of the system can be expanded around $\left\{\phi_{n}^{0}\right\}$. If the higherorder terms (above the second-order term) are omitted, the total energy of the system can be written as

$$
\begin{aligned}
H= & H_{e}+H_{\text {elastic }} \\
= & E_{0}\left(\left\{\phi_{n}^{0}\right\}\right)+\sum_{m} A_{m}\left(\left\{\phi_{n}^{0}\right\}\right) \delta \phi_{m} \\
& +\frac{1}{2} \sum_{m, n} B_{m n}\left(\left\{\phi_{n}^{0}\right\}\right) \delta \phi_{m} \delta \phi_{n} .
\end{aligned}
$$

$A_{m} \equiv 0$ corresponds to the lowest energy state, thus, we obtained the self-consistent equation

$$
(-1)^{n}\left(\phi_{n}+\phi_{n+1}\right)=\frac{2 \alpha}{K} \sum_{\mu}^{\text {occ }} Z_{n, \mu} Z_{n+1, \mu}+\frac{K^{\prime}}{K},
$$

where the energies $\varepsilon_{\mu}$ and the wave function $\mathrm{Z}_{n, \mu}$ of electrons are determined by the electronic eigenequation

$$
H_{e} \sum_{n} Z_{n, \mu}|n\rangle=\varepsilon_{\mu} \sum_{n} Z_{n, \mu}|n\rangle
$$

Since the electron-electron interactions are considered, the electronic eigenequation should also be solved selfconsistently. In the approximation of Hartree-Fock

$$
\begin{aligned}
\sum_{n, s} C_{n, s}^{+} C_{n, s} C_{n,-s}^{+} C_{n,-s} & \\
\rightarrow & \sum_{n, s}\left(\chi_{n, s} C_{n, s}^{+} C_{n, s}+\chi_{n,-s} C_{n,-s}^{+} C_{n,-s}\right) \\
& -\sum_{n, s} \chi_{n, s} \chi_{n,-s},
\end{aligned}
$$

where $\chi_{n, s}=\left\langle\Phi\left|C_{n,-s}^{+} C_{n,-s}\right| \Phi\right\rangle$. State $\Phi$, which is used to calculate the average value, is the ground state of selfconsistent field, and it should also be determined selfconsistently. It means that the electronic eigenequation should be self-consistently solved.

With any initial configuration as input, the eigenenergies and wave functions can be obtained by solving selfconsistently the electronic eigenequation. Then using the calculated eigenenergies and wave functions, we can obtain a new configuration from the self-consistent equation. With the new configuration as input, we can get new eigenenergies and wave functions by solving the electronic eigenequation self-consistently again and then solving the self-consistent equation again to get new configurations, etc. Iterating in this way until the configuration difference of the two successive iterations reaches a certain precision (e.g., less than ten minus seven), we can self-consistently obtain the solutions of atomic configurations, electronic eigenvalues, electronic wave functions, and the total energy at the lowest energy states of the system in the presence of any external field.

In the calculation $t_{0}=2.5 \mathrm{eV}, K=21 \mathrm{eV} / \AA^{2}, \alpha=4.1$ $\mathrm{eV} / \AA \cap, \quad N=100$, and $K^{\prime}=-1.25032 \alpha$ are used, ${ }^{16}$ the symmetry-breaking term $t_{e}$ changes from 0 to $0.1 \mathrm{eV}$, as for the electron-electron interactions, $U$ ranges from $0-4 \mathrm{eV}$, and $V \leqslant U / 2$. Our calculations show that the change of the parameters only affects the concrete numerical values, and it will not change the basic physics.

\section{MAIN RESULTS}

\section{A. The self-trapping excited states of conjugated polymers are formed in the presence of electric fields}

Extra electron(s) and hole(s) in conjugated polymers exist since the applied electric field injects electrons and holes into the active layer of conjugated polymers based light-emitting devices. Using the method mentioned above, we obtained the lowest energy states in the following three different cases: (1) the ground state, (2) the single-excited state [a hole at the highest occupied molecule orbit (HOMO) and an electron at the lowest unoccupied molecule orbit (LUMO) exist], (3) the biexcited state (there are two holes at the HOMO level and two electrons at the LUMO level). In these three different 


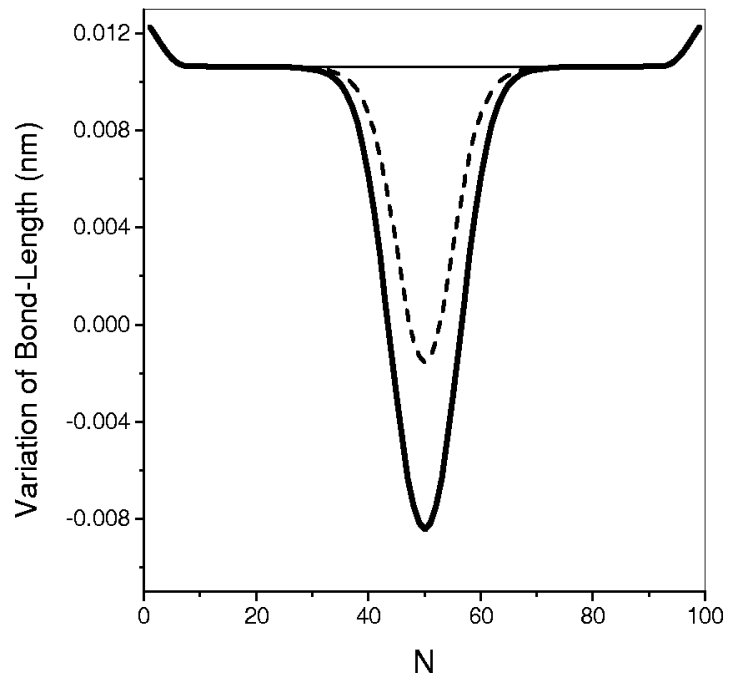

FIG. 1. The variation of bond length along a finite chain for three different cases: the thin line represents the ground state, the dashed line is the single-excited state (to excite one electron), the thick line denotes the biexcited state (to excite two electron). The electric field $E$ is in the range of 0 to $5 \times 10^{5} \mathrm{~V} / \mathrm{cm}$.

cases, the variations of the bond length along a finite chain are depicted in Fig. 1. Figure 1 shows that the extra elec$\operatorname{tron}(\mathrm{s})$ and hole(s) induce the local deformation of the lattice, leading to a strained field. In the meantime, the extra electron(s) and hole(s) themselves are trapped by the strain field, ${ }^{1-15}$ and the self-trapping bound state of electron(s)hole(s) is formed. The bound state of the electron(s) and hole(s) is also known as the exciton. So the self-trapping single-excited states can also be called self-trapping single excitons or bipolaron single excitons ${ }^{11-14}$ and the selftrapping biexcited states, self-trapping biexcitons, or bipolaron biexcitons. ${ }^{13,15}$

The formation of the self-trapping single(bi)-excited state not only induces lattice distortion but also affects the electronic states. As a result, the HOMO and LUMO levels enter the original energy gap and become the localized deep levels $\varepsilon_{\text {low }}$ and $\varepsilon_{\text {high }}$. The low level $\varepsilon_{\text {low }}$ is the bonding localized state with the energy lower than the gap center. The high level $\varepsilon_{\text {high }}$ is the antibonding localized state with the energy higher than the gap center. The schematic diagram of energy levels for the self-trapping excited states is shown in Fig. 2, in which (a) is for a self-trapping single-excited state since there is an extra hole at the $\varepsilon_{\text {low }}$ level and an extra electron at the $\varepsilon_{\text {high }}$ level and (b) is for a self-trapping biexcited state since there are two extra holes at the $\varepsilon_{\text {low }}$ level and two extra electrons at the $\varepsilon_{\text {high }}$ level.

Our calculations show that for given $t_{e}$, order parameter $\phi_{n}$ versus $N$ does not change for the ground state, the singleexcited state and the biexcited state in the field range of 0 to $5 \times 10^{5} \mathrm{~V} / \mathrm{cm}$, as shown in Fig. 1 .

\section{B. Weak or moderate fields polarized the self-trapping excited states}

The charge density is

$$
\rho=\sum_{\mu, s}^{\text {occ }}\left|Z_{n, \mu}^{s}\right|^{2} .
$$

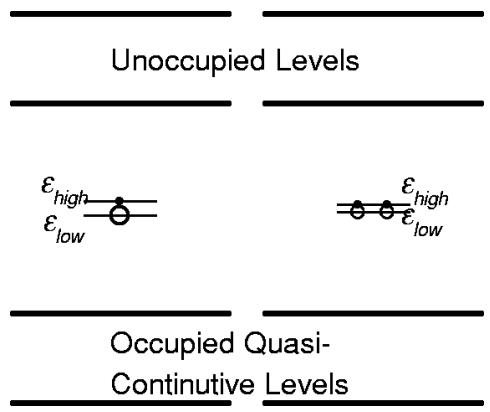

FIG. 2. The schematic diagram of the energy levels for the self-trapping excited states in polymers. The HOMO level is $\varepsilon_{\text {low }}$ level and LUMO level is $\varepsilon_{\text {high }}$ level. (a) The single-excited state (to excite one electrons, so there are one hole at the HOMO level and one electron at the LUMO level). (b) The biexcited state (to excite two electrons, so there are two holes at the HOMO level and two electrons at the LUMO level).

When there is no external field, the charge density distributions of the single-excited state and the biexcited state are the same as that of the ground state, i.e., everywhere is electrically neutral. This implies that the distribution of the electron and hole are the same within the self-trapping excited state of conjugated polymers, when no external field exists.

After applying an electric field, it is found that the electric field transfers some of the charges in a self-trapping excited state. As a result, the distribution of the charge density is changed, and the self-trapping excited state is polarized. The degree of polarization increases linearly with the electric field $E$ at weak or moderate fields.

The charge density distribution of the self-trapping singleexcited and biexcited states is shown, respectively, in Figs. 3 and 4, where the unit of the ordinate is the value of an electron charge $e$ and an electric field of $E=10^{5} \mathrm{~V} / \mathrm{cm}$ is along the chain direction. In these figures, the charge density of the ground state in the same electric field has been subtracted.

Figure 3 shows that in the self-trapping single-excited state, the positive charges move along the electric field and

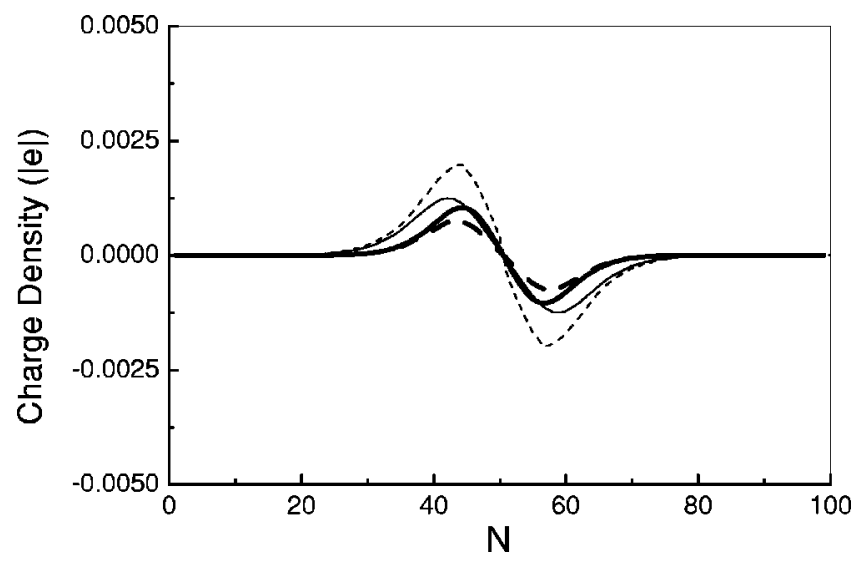

FIG. 3. The charge density distribution of the self-trapping single-excited state in the field $E=10^{5} \mathrm{~V} / \mathrm{cm}$. $E$ is along the chain direction. The unit of the ordinate is the value of an electron charge $e$. The thin solid line is calculated using $U=0$ and $V=0$, the thin dashed line using $U=4 \mathrm{eV}$ and $V=0$, the thick solid line using $U=4.0 \mathrm{eV}$ and $V=0.6 \mathrm{eV}$, the thick dashed line using $U=2 \mathrm{eV}$ and $V=0.6 \mathrm{eV}$. 


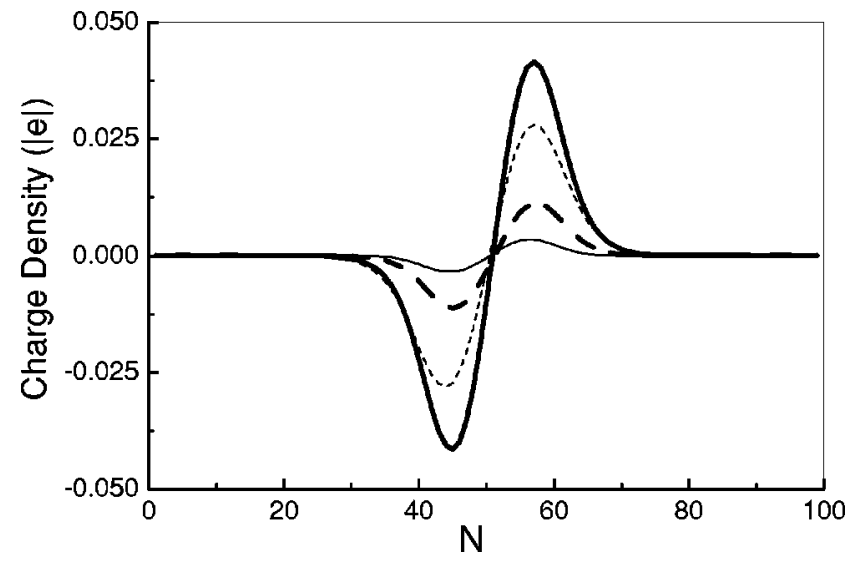

FIG. 4. The charge density distribution of the self-trapping biexcited state in the field $E=10^{5} \mathrm{~V} / \mathrm{cm}$. $E$ is along the chain direction. The unit of the ordinate is the value of an electron charge $e$. The thin solid line is calculated using $U=0$ and $V=0$, the thin dashed line using $U=4 \mathrm{eV}$ and $V=0$, the thick solid line using $U=4.0 \mathrm{eV}$ and $V=0.6 \mathrm{eV}$, the thick dashed line using $U=2 \mathrm{eV}$ and $V=0.6 \mathrm{eV}$.

the negative charges drift in the opposite direction. This is normal polarization. Figure 3 also shows that a singleexcited state in conjugated polymers displays weak normal polarization.

It should be noted that the self-trapping biexcited state exhibits the behavior of negative polarization, i.e., the positive charges move in the opposite direction of the field and the negative charges along the field. Figure 4 demonstrates that a self-trapping biexcited state in conjugated polymers displays obvious negative polarization. The ordinate of Fig. 4 is ten times as large as that of Fig. 3.

In Figs. 3 and 4, the thin solid line is calculated using $U=0$ and $V=0$, the thin dashed line using $U=4 \mathrm{eV}$ and $V=0$. By comparing the thin solid and dashed lines in Fig. 3 or 4, respectively, it is clear that the on-site Hubbard energy $U$ obviously increases the polarization of self-trapping excited states. In these two figures, the thick solid line is calculated using $U=4.0 \mathrm{eV}$ and $V=0.6 \mathrm{eV}$, the thick dashed line using $U=2 \mathrm{eV}$ and $V=0.6 \mathrm{eV}$. Comparing the thick solid and dashed lines in Fig. 3 or 4, we can see that the nearest neighbor Coulomb interaction $V$ obviously increases the negative polarization of a self-trapping biexcited state in conjugated polymers, however, the interaction $V$ restrains the normal polarization of a self-trapping single-excited state in conjugated polymers slightly. In addition, our calculations also show that the normal polarization of a self-trapping single-excited state is not sensitive to $t_{e}$, whereas the negative polarization of a self-trapping biexcited state increases rapidly as $t_{e}$ decreases.

\section{Strong fields transfer all the charges in the self-trapping excited states}

Strong fields will transfer all the charges in the selftrapping excited states of conjugated polymers. It is observed that a critical field $E_{\text {critical }}$ exists which will induce a sudden change of both bond length and charge density as well as polarizability. Figure 5 represents the charge density distribution [Fig. 5(a)] and the variations of bond length along a

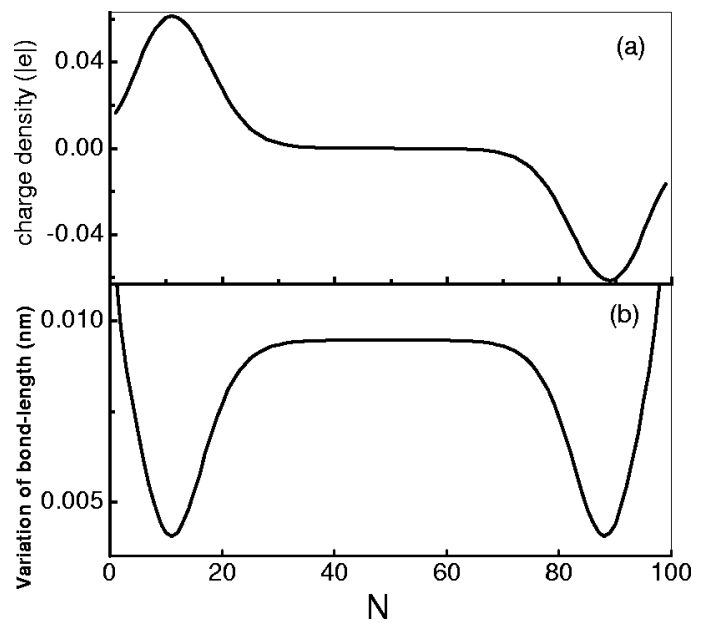

FIG. 5. The self-trapping single-excited state for $t_{e}=0.06 \mathrm{eV}$ has been dissociated into a hole polaron and an electron polaron at the critical electric field $E=9.6 \times 10^{5} \mathrm{~V} / \mathrm{cm}$, and $U=1.0 \mathrm{eV}, V$ $=0$. (a) The charge density distribution and (b) the variation of bond length along a finite chain.

finite chain [Fig. 5(b)] at $E \geqslant E_{\text {critical }}$. Figure 5(a) shows that two charges have parted by a strong electric field. From Fig. 5 (a), it can be confirmed that the charge quantity in the right peak is $+e$ and that in the left one it is $-e$. Figure $5(\mathrm{~b})$ illustrates that two polarons exist along a finite chain when $E \geqslant E_{\text {critical }}$. By combining Figs. 5(a) and 5(b), it is obvious that the left polaron in Fig. 5 is an electron polaron and the right one is a hole polaron.

Figure 5 can be explained using the following arguments. The self-trapping single-excited state is in fact a bipolaron single exciton, as mentioned above. When $E=0$, the energy to create a bipolaron single exciton is less than that to create an electron polaron plus a hole polaron. ${ }^{8}$ Let the energy difference be $\Delta(0)$. So at $E=0$, a bipolaron single-exciton is the lowest-energy state for a polymer chain with an extra electron-hole pair. After an electric field is applied, the field will decrease the energy of an electron polaron plus a hole polaron, and the decreased energy $\Delta(E)=e E N a$ ( $a$ is the lattice constant, $N a$ is the length of the chain, $E$ is the electric field). Obviously, a critical field exists when an electric field is so strong that $\Delta(E) \geqslant \Delta(0)$ [if $E=E_{\text {critical }}, \Delta(E)$ $=\Delta(0)]$, the energy of the charged-polaron pair will be less than that of a bipolaron single exciton. So at strong fields, a bipolaron single exciton is unstable, and the energy of the well-separate charged-polaron pair will be less than that of a bipolaron single exciton. Therefore, the lowest-energy state is to be an electron polaron plus a hole polaron at strong fields, as shown in Fig. 5. Hence, an electron polaron plus a hole polaron at a strong field is a successor of a bipolaron single exciton. In other words, a strong field is bound to split a bipolaron single exciton directly into an electron polaron plus a hole polaron. This result is consistent with the recent field-assisted experiments performed on polymeric light emitting devices, ${ }^{1}$ in which it was found that charged polarons are formed from exciton fission without intermediate states in the conjugated polymer.

After an exciton is split into a charged-polaron pair, the two charged polarons will move in opposite directions in an applied electric field and are well separated. As a result, they have little chance to recombine. So the split of the self- 


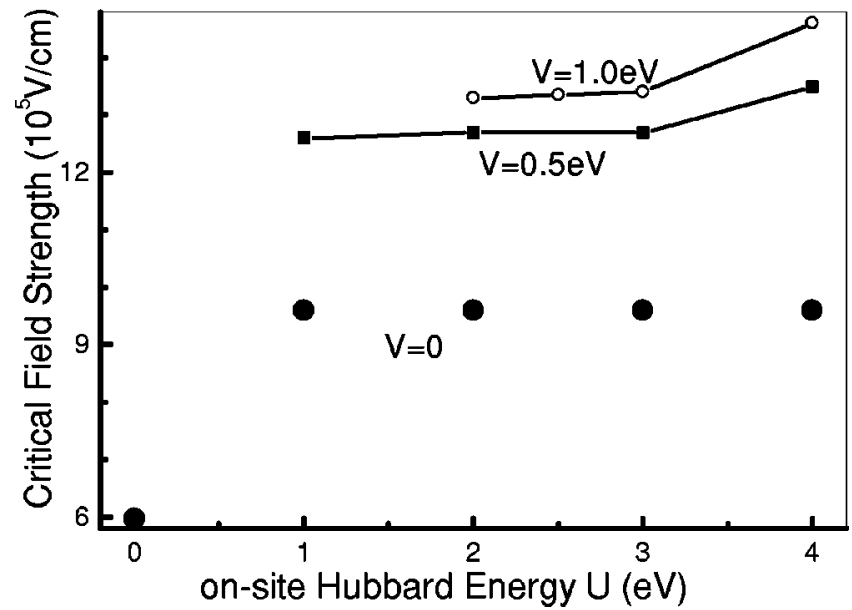

FIG. 6. The critical electric field versus on-site Hubbard energy $U$ at different nearest neighbor Coulomb interaction $V$. The solid circle represents $V=0$, the solid square is $V=0.5 \mathrm{eV}$, the open circle denotes $V=1.0 \mathrm{eV}$.

trapping single-excited states (emitting species) in conjugated polymers leads to PL quenching. The result is consistent with experiments ${ }^{2-6}$ in which field-induced PL quenching was observed. Since the split of a bipolaron exciton depends only on the electrical field along the polymer chain, and the orientations of polymer chains are randomly distributed, PL will not be quenched in those polymer chains which are not along the field when $E=E_{\text {critical }}$. When the applied electric field is stronger than $E_{\text {critical }}$, the number of polymer chains, along which the field projection is $\geqslant E_{\text {critical }}$, increases. This explains why the PL intensity decreases with the increase of the applied field. ${ }^{2-5}$ Some polymer chains are almost perpendicular to the field, so that along these chains the field projection is less than $E_{\text {critical }}$ even at very high field. This explains why the PL intensity will not be quenched completely, as the applied field is further increased. ${ }^{2-5}$

Electron-electron interactions have a strong impact on the abovementioned splitting into polaronic states. The critical electric field versus on-site Hubbard energy $U$ at different nearest neighbor Coulomb interactions $V$ is depicted in Fig. 6 , in which the solid circle represents $V=0$, the solid square is $V=0.5 \mathrm{eV}$, and the open circle denotes $V=1.0 \mathrm{eV}$. Figure 6 reveals that the stronger the electron-electron interaction, the larger the critical field. The reason for this is that the electron hole interaction in a self-trapping excited state increases with the electron-electron interaction, as does the binding energy of the electron and hole in a self-trapping excited state. As a result, a stronger critical field is needed to split a self-trapping excited state when the electron-electron interactions are stronger.

Our calculations also show that when $t_{e}$ decreases, the critical field tends to increase. For example, when $t_{e}=0.1$ $\mathrm{eV}, E_{\text {critical }}=4.9 \times 10^{5} \mathrm{~V} / \mathrm{cm}$; when $t_{e}=0.06 \mathrm{eV}, E_{\text {critical }}$ $=5.97 \times 10^{5} \mathrm{~V} / \mathrm{cm}$; and when $t_{e}=0.05 \mathrm{eV}, E_{\text {critical }}=7.83$ $\times 10^{5} \mathrm{~V} / \mathrm{cm}$ at $U=0$ and $V=0$. To avoid the field dissociation of the single-excited state, the strength of the electric field we use is not larger than $2 \times 10^{5} \mathrm{~V} / \mathrm{cm}$ when we discuss the polarization of self-trapping excited states in conjugated polymers.

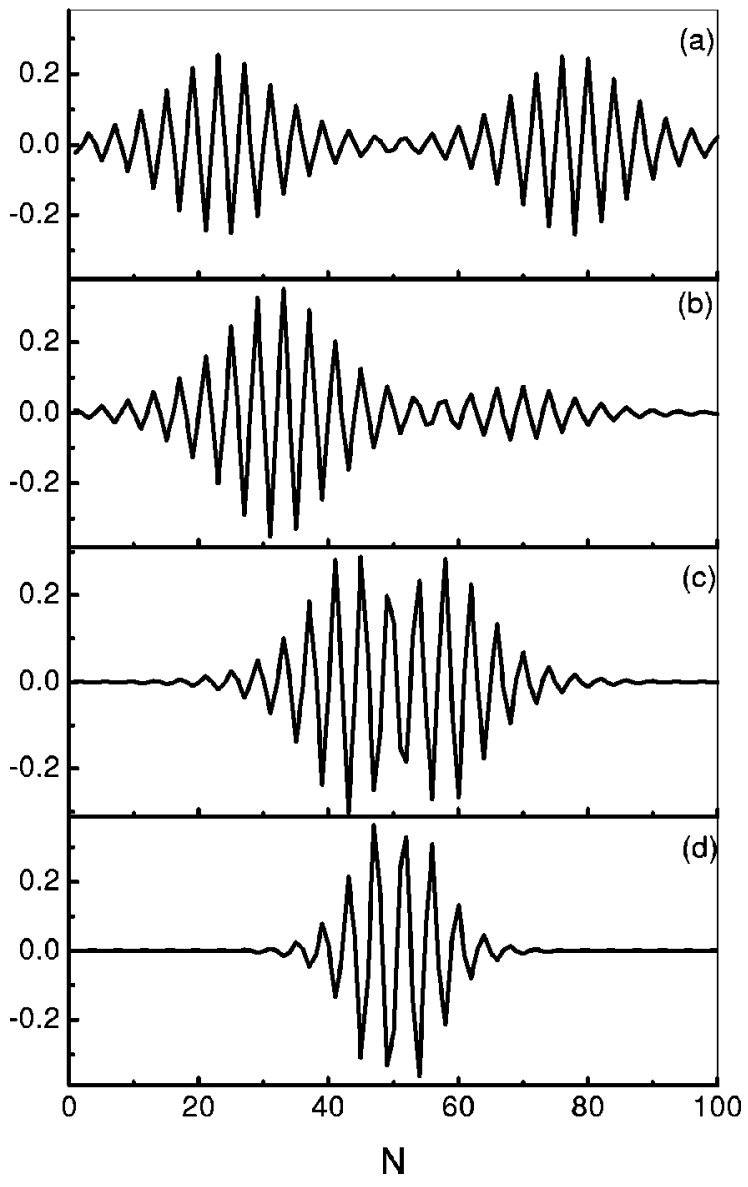

FIG. 7. Wave function corresponding to $\varepsilon_{\text {low }}$ level. The electric field is along the chain direction. (a) $t_{e}=0, E=0$, (b) $t_{e}=0, E$ $=10^{5} \mathrm{~V} / \mathrm{cm}$, (c) $t_{e}=0.05 \mathrm{eV}, E=10^{5} \mathrm{~V} / \mathrm{cm}$, (d) $t_{e}=0.1 \mathrm{eV}, E$ $=10^{5} \mathrm{~V} / \mathrm{cm}$.

\section{The polarization characteristics of the localized electronic states close to the midgap in the self-trapping excited states}

In order to disclose the physical origin of the polarization characteristics described in Figs. 3 and 4, we analyzed the wave functions of the electronic states, since the charge density is equal to a sum of squares for the absolute values of the wave functions of all the occupied states. We found that the wave functions of a single-excited state are very similar to those of a biexcited state, and their variations with the electric field are very similar too. In addition, we noted that the difference between a single-excited state and a biexcited state in conjugated polymers is the different occupations of electrons on the two localized levels close to the midgap: for the single-excited state, there is an electron in both $\varepsilon_{\text {low }}$ and $\varepsilon_{\text {high }}$ levels, whereas, for the biexcited state, there is no electron in the $\varepsilon_{\text {low }}$ level and there are two electrons in the $\varepsilon_{\text {high }}$ level, as depicted in Figs. 2(a) and 2(b). Hence, we studied the effects of the electric field on the wave functions corresponding to the two localized states ( $\varepsilon_{\text {low }}$ and $\varepsilon_{\text {high }}$ states).

As mentioned above, in a self-trapping single(bi)-excited state, two localized electronic states (i.e., localized levels) exist close to the midgap: the low level $\left(\varepsilon_{\text {low }}\right)$ and the high level $\left(\varepsilon_{\text {high }}\right)$. Figures 7 and 8 depict the wave function corresponding to $\varepsilon_{\text {low }}$ and $\varepsilon_{\text {high }}$ levels, respectively. In the two figures, (a) is obtained with $t_{e}=0, E=0$, two wave packets 


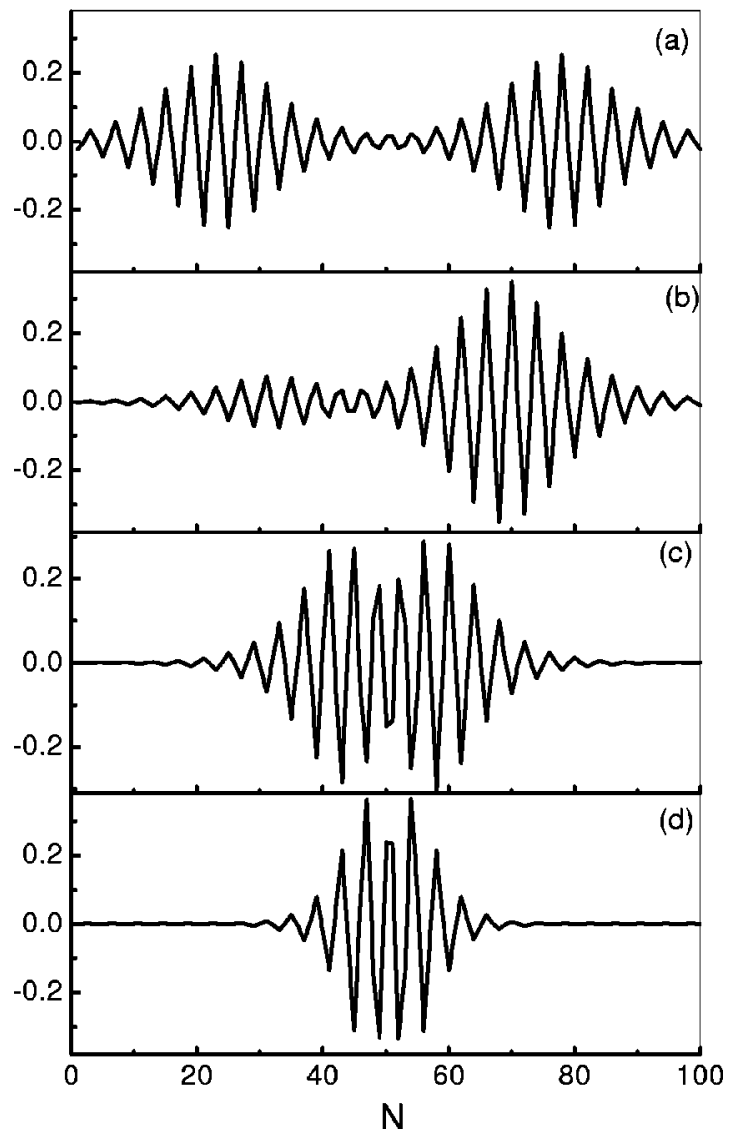

FIG. 8. Wave function corresponding to $\varepsilon_{\text {high }}$ level. The electric field is along the chain direction. (a) $t_{e}=0, E=0$, (b) $t_{e}=0, E$ $=10^{5} \mathrm{~V} / \mathrm{cm},(\mathrm{c}) t_{e}=0.05 \mathrm{eV}, E=10^{5} \mathrm{~V} / \mathrm{cm}$, (d) $t_{e}=0.1 \mathrm{eV}, E$ $=10^{5} \mathrm{~V} / \mathrm{cm}$.

in the figure express that two electrons can be accommodated in one level, (b) with $t_{e}=0, E=10^{5} \mathrm{~V} / \mathrm{cm}$, (c) with $t_{e}$ $=0.05 \mathrm{eV}, E=10^{5} \mathrm{~V} / \mathrm{cm}$, and (d) with $t_{e}=0.1 \mathrm{eV}, E=10^{5}$ $\mathrm{V} / \mathrm{cm}$. Comparing (a) and (b), we can very clearly see that under the electric field, the wave function of the low level $\left(\varepsilon_{\text {low }}\right)$ corresponds to normal polarization (i.e., electrons move in the opposite direction of the electric field), and that of the high level ( $\varepsilon_{\text {high }}$ ) to negative polarization (i.e., electrons transfer along the electric field).

It is important to understand the reason why the wave function of the low level $\left(\varepsilon_{\text {low }}\right)$ corresponds to normal polarization, whereas the wave fuction of the high level $\left(\varepsilon_{\text {high }}\right)$ corresponds to negative polarization. This can be explained qualitatively as follows on the basis of the quantummechanics theory of polarization.

The Hamiltonian in an electric field is

$$
H=H_{0}+H^{\prime} .
$$

$H^{\prime}$, the perturbation induced by the electric field, can be written as

$$
H^{\prime}=-P E,
$$

where $P$ is the polarized vector of the system and $P$ $=-e \int \rho(x) x d x$. Since

$$
H_{0} \psi_{\mu}^{0}=\varepsilon_{\mu}^{0} \psi_{\mu}^{0}, \quad H \psi_{\mu}=\varepsilon_{\mu} \psi_{\mu},
$$

the wave function including the first order of $H^{\prime}$ is

$$
\psi_{\mu}=\psi_{\mu}^{0}+\sum_{\nu}{ }^{\prime} \frac{H_{\mu \nu}^{\prime}}{\varepsilon_{\mu}-\varepsilon_{\nu}} \psi_{\mu}^{0},
$$

where the summation $\Sigma_{\nu}^{\prime}$ excludes $\mu=\nu$. Since $H_{\mu \nu}^{\prime}$ $=-P_{\mu \nu} E$,

$$
\psi_{\mu}=\psi_{\mu}^{0}-\sum_{\nu}{ }^{\prime} \frac{P_{\mu \nu} E}{\varepsilon_{\mu}-\varepsilon_{\nu}} \psi_{\nu}^{0} .
$$

The polarization of the $\mu$ th level can be expressed as

$$
\begin{aligned}
P_{\mu} & =\left\langle\psi_{\mu}|P| \psi_{\mu}\right\rangle \\
& =\left\langle\psi_{\mu}^{0}|P| \psi_{\mu}^{0}\right\rangle-E \sum_{\nu}{ }^{\prime} \frac{2\left|P_{\mu \nu}\right|}{\varepsilon_{\mu}-\varepsilon_{\nu}}+\text { higher-order terms, }
\end{aligned}
$$

where the first term corresponds to zero field and the second term is due to the polarization induced by the electric field. Since $\Delta P=\chi E$, the polarizability of the $\mu$ th level is

$$
\chi_{\mu}=\sum_{\nu}{ }^{\prime} \frac{2\left|P_{\mu \nu}\right|^{2}}{\varepsilon_{\mu}-\varepsilon_{\nu}} .
$$

The two localized levels $\varepsilon_{\text {low }}$ and $\varepsilon_{\text {high }}$ are energetically close to each other, and far from the other levels. According to the distribution of energy, the $\varepsilon_{\text {high }}$ level dominates the contributions to $\chi_{\text {low }}$, as does the $\varepsilon_{\text {low }}$ level to $\chi_{\text {high }}$, and the contribution of the other levels to $\chi_{\text {low }}$ or $\chi_{\text {high }}$ can be neglected. Furthermore, the wave function of the $\varepsilon_{\text {low }}$ level has odd parity and the wave function of the $\varepsilon_{\text {high }}$ level has even parity. Since the two (odd and even) wave functions completely "'match,' their dipole transition matrix element is large and makes dominant contributions to $P$, while the wave functions of the other levels do not match, the dipole matrix element of the other levels is thus small and contributes little to $P$. Because of these reasons, $\chi_{\text {low }}$ and $\chi_{\text {high }}$ can be written approximately as

$$
\begin{aligned}
& \chi_{\mathrm{low}} \simeq \frac{2|P|^{2}}{\varepsilon_{\mathrm{low}}-\varepsilon_{\mathrm{high}}}, \\
& \chi_{\mathrm{high}} \simeq \frac{2|P|^{2}}{\varepsilon_{\mathrm{high}}-\varepsilon_{\mathrm{low}}} .
\end{aligned}
$$

Since $\varepsilon_{\text {low }}<\varepsilon_{\text {high }}, \chi_{\text {low }}<0$, i.e., the electron on the low level $\left(\varepsilon_{\text {low }}\right)$ moves in the opposite direction of the electric field (normal polarization). However, $\chi_{\text {high }}>0$, i.e., the electron on the high level ( $\left.\varepsilon_{\text {high }}\right)$ drifts along the the electric field (negative polarization). Hence, the direction of the polarization of these two localized electronic states is opposite.

It can also be qualitatively explained why the polarization of these two localized electronic states decreases quickly as $t_{e}$ increases as follows.

(1) Comparing (a), (c), and (d) in Figs. 7 and 8, we become aware that for larger $t_{e}$, the two "wave packets" of the wave function corresponding to the localized level $\varepsilon_{\text {low }}$ or $\varepsilon_{\text {high }}$ are more strongly overlapped. Thus it is more difficult to polarize the wave function as $t_{e}$ increases. Therefore, the polarization of the two localized states decreases with $t_{e}$. 


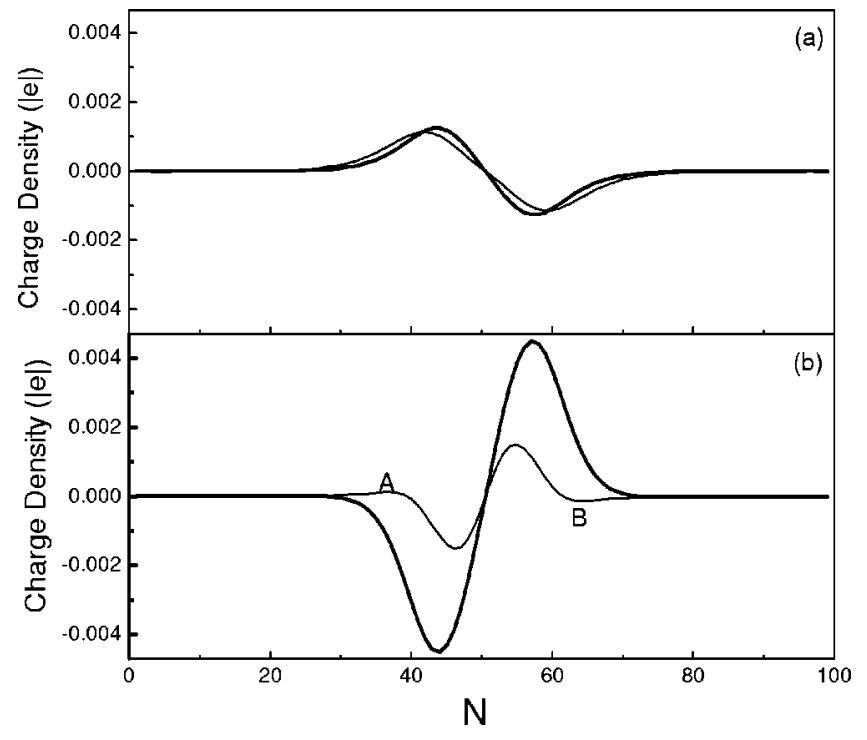

FIG. 9. The charge density distribution of the self-trapping excited states with different $t_{e}$ in the field $E$. The thick line was calculated using $t_{e}=0.05 \mathrm{eV}$, while the thin line using $t_{e}=0.1 \mathrm{eV}$. $E=10^{5} \mathrm{~V} / \mathrm{cm}$, and $E$ is along the chain direction. (a) Self-trapping single-excited state and (b) self-trapping biexcited state.

(2) The larger $t_{e}$ is, the more localized the states are. Accordingly, the amplitude of the wave function corresponding to the localized level $\varepsilon_{\text {low }}$ or $\varepsilon_{\text {high }}$ approaches zero at a lot of lattice points. As a result, the dipole matrix element will be small. Hence, the polarizability also decreases as $t_{e}$ increases. Due to these two reasons, the polarization of these two localized electronic states decrease quickly with the increase of $t_{e}$.

\section{E. The physical origin on the polarization of the self-trapping excited states in conjugate polymers}

Two different macroscopic polarization characteristics in a conjugate polymer with extra electron(s) and hole(s) can be illustrated as follows. For a self-trapping single-excited state, there is only one electron on both the $\varepsilon_{\text {low }}$ (the 50th level in the article) and $\varepsilon_{\text {high }}$ (the 51st level in the article) levels. In addition, the quasicontinuative levels (the 1st-49th levels in the article) are fully occupied. The negative polarization of the $\varepsilon_{\text {high }}$ level is offset by the normal polarization of the $\varepsilon_{\text {low }}$ level. Therefore, the normal polarization of the singleexcited state originates from the fully occupied quasicontinuative levels. This also means that the fully occupied quasicontinuative levels display the characteristic of small normal polarization (as shown in Fig. 3). Also the polarization of the occupied quasicontinuative levels changes very little with $t_{e}$, as described in Fig. 9(a).

For a self-trapping biexcited state, there are two electrons on the $\varepsilon_{\text {high }}$ level (the 51th level in the article) and there is no electron on the $\varepsilon_{\text {low }}$ level (the 50th level in the article). In addition, the quasicontinuative levels (the 1st-49th levels in the article) are also fully occupied. So the polarization of a self-trapping biexcited state should equal the difference between the negative polarization of the occupied $\varepsilon_{\text {high }}$ level and the normal polarization of the fully occupied quasicontinuative levels. As a result, the biexcited state shows obvi- ous negative polarization (as depicted in Fig. 4) due to the large negative polarization of the $\varepsilon_{\text {high }}$ level.

The polarization of the localized electronic state decreases quickly with $t_{e}$, while the polarization of the occupied quasicontinuative levels weakly depends on $t_{e}$. This leads to the quick decrease of the negative polarization of a self-trapping biexcited state with $t_{e}$, as described in Fig. 9(b). Taking a close look at the thin solid curve $\left(t_{e}=0.1 \mathrm{eV}\right)$ in Fig. 9(b), one can see that region A (accumulation with a small amount of negative charge) and region B (accumulation with a small amount of positive charge) exist near the region of the negative polarization. Regions A and B are just the remainders of the normal polarization induced by the quasicontinuative levels which have not been completely cancelled by the negative polarization originated from the occupied $\varepsilon_{\text {high }}$ localized level. So the self-trapping biexcited state displays quite small negative polarization when $t_{e}=0.1 \mathrm{eV}$.

When $t_{e} \geqslant 0.2 \mathrm{eV}$, the negative polarization of the occupied $\varepsilon_{\text {high }}$ level is a little bit weaker than the normal polarization induced by the occupied quasicontinuative levels. Therefore, the self-trapping biexcited state shows weak normal polarization when $t_{e} \geqslant 0.2 \mathrm{eV}$.

In addition, the charge transfer quantity of the localized electronic states increases quickly as the electron-electron interactions $U$ and $V$ increase, whereas the total charge transfer quantity of the occupied quasicontinuative levels is affected slightly by $U$ and $V$. As a result, the electron-electron interactions obviously increase the negative polarization of a self-trapping biexcited state, as shown in Fig. 4.

\section{DISCUSSION}

We have demonstrated that the macroscopic negative polarization of a self-trapping biexcited state is originated from the negative polarization of the microscopic electronic state. It is well known that electronic states have orthogonality. The orthogonality of the wave functions might imply that it is impossible that all the electronic states have normal polarization in the presence of an electric field. The orthogonality of the wave functions will compel some microscopic electronic states having negative polarization.

From the viewpoint of energy, the electronic state which has negative polarization has higher energy, whereas the electronic state with normal polarization has lower energy. Hence, if the occupied states are quasicontinuative (here "quasicontinuative" means that there is no unoccupied state under the highest occupied state), no negative polarization exists. Only when there is unoccupied state under the highest occupied state could there be macroscopic negative polarization (the condition being that the negative polarization of the occupied state, which is above the unoccupied state, is larger than the normal polarization of the quasicontinuative occupied states, which are under the unoccupied state). Therefore, only an excited state may have negative polarization.

Then what are the predictions of the model for the relative contributions of the polarization of single excitons and biexcitons, and at what concentration of biexcitons can we observe negative polarization? In a realistic system, both single excitons and biexcitons contribute to the average polarization, which depends not only upon the ratio of the concentrations for these two types of excitations, but also upon the 


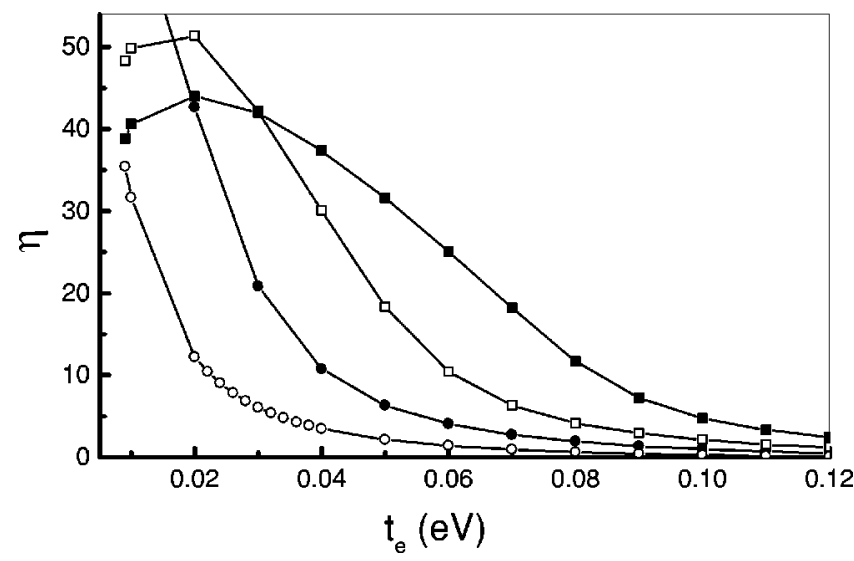

FIG. 10. Symmetry-breaking term $t_{e}$ dependence of $\eta$ under different electron interactions $U$ and $V . \eta$ is the absolute value of the ratio between the polarization of a biexciton and the polarization of a single exciton. The open circle is calculated using $U=0$ and $V=0$, the solid circle using $U=1.0 \mathrm{eV}$ and $V=0.5 \mathrm{eV}$, the open square using $U=2.0 \mathrm{eV}$ and $V=0.5 \mathrm{eV}$, the solid square using $U$ $=3.0 \mathrm{eV}$ and $V=0.5 \mathrm{eV}$, and the electric field in the calculations is $E=10^{5} \mathrm{~V} / \mathrm{cm}$.

ratio between the polarization of a biexciton and the polarization of a single exciton. The ratio of the concentrations is sensitive to the intensity of photoexcitation in photoconductivity and/or photoluminescence type experiments or to the injection level in the study of electroluminescence, whereas the ratio between the polarization of a biexciton and the polarization of a single exciton is affected greatly by the symmetry-breaking term $t_{e}$ and electron interactions $U$ and $V$, as discussed in Secs. III B, III D, and III E. The absolute value $\eta$ of the ratio between the polarization of a biexciton and the polarization of a single exciton versus the symmetrybreaking term $t_{e}$ under different electron interactions is depicted in Fig. 10.

Let the concentration of biexcitons be $N_{0}$ [and the concentration of single excitons be $\left.\left(1-N_{0}\right)\right]$, when the negative polarization $P_{\mathrm{bi}}$ of biexcitons is just offset by the normal polarization $P_{\text {single }}$ of single excitons. This means that $P_{\mathrm{bi}}$ $N_{0}=P_{\text {single }} \quad\left(1-N_{0}\right)$. So $N_{0}=1 /(1+\eta)$, since $\eta$ $=P_{\mathrm{bi}} / P_{\text {single }}$. When the concentration of biexcitons in a polymer system is larger than $N_{0}$, the system will show negative polarization. Figure 11 shows $N_{0}$ versus $t_{e}$ under different electron interactions. Figure 11 tells us that $N_{0}$ is affected easily by $t_{e}, U$, and $V$. For example, if $t_{e}=0.06 \mathrm{eV}$, $N_{0}=0.418$ when $U=0, V=0 ; N_{0}=0.198$ when $U=1.0 \mathrm{eV}$, $V=0.5 \mathrm{eV} ; N_{0}=0.088$ when $U=2.0 \mathrm{eV}, V=0.5 \mathrm{eV}$; however, if $t_{e}=0.12 \mathrm{eV}, N_{0}=0.963$ when $U=0, V=0 ; N_{0}$ $=0.678$ when $U=1.0 \mathrm{eV}, V=0.5 \mathrm{eV} ; N_{0}=0.461$ when $U$ $=2.0 \mathrm{eV}, V=0.5 \mathrm{eV}$. When the concentration of biexcitons is larger than $N_{0}$, the polymer system may show negative polarization (i.e., negative polarization will emerge when the concentration of biexcitons is above the corresponding curve of Fig. 11. In other words, negative polarization is above the curve, whereas, normal polarization is below the curve of Fig. 11. Hence in order to get negative polarization, the polymer material with small symmetry-breaking term $t_{e}$ and large electron interactions $U$ and $V$ should be employed.

The symmetry-breaking term $t_{e}$ is the energy difference between phase $\mathrm{A}$ and phase $\mathrm{B}$ of the nondegenerate ground

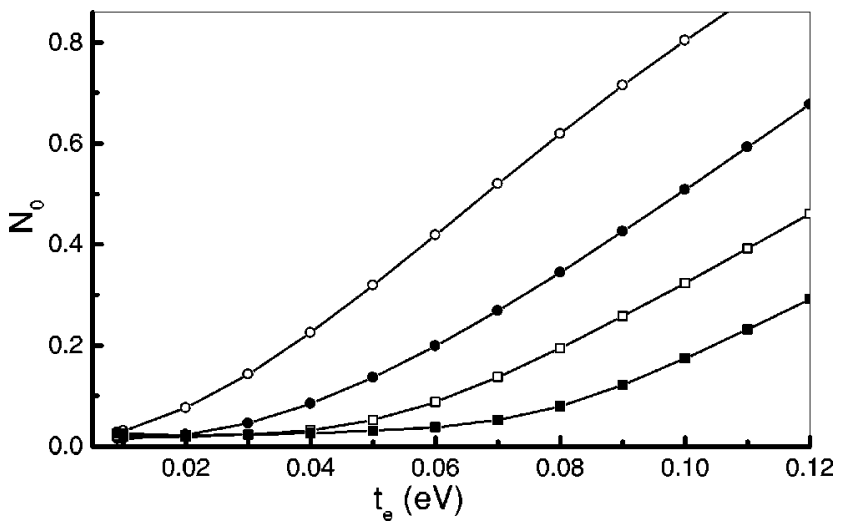

FIG. 11. $N_{0}$ versus $t_{e}$ under different electron interactions $U$ and $V . N_{0}$ is the concentration of biexcitons when the negative polarization of biexcitons is just offset by the normal polarization of single excitons. The open circle is calculated using $U=0$ and $V$ $=0$, the solid circle using $U=1.0 \mathrm{eV}$ and $V=0.5 \mathrm{eV}$, the open square using $U=2.0 \mathrm{eV}$ and $V=0.5 \mathrm{eV}$, the solid square using $U$ $=3.0 \mathrm{eV}$ and $V=0.5 \mathrm{eV}$, and the electric field in the calculations is $E=10^{5} \mathrm{~V} / \mathrm{cm}$.

states for each bond. In polyparaphenylene (PPP), polythiophene (PT), and polypyrrole (PPy), the energy difference between quinoid (phase B) and benzenoid (phase A) is reported to be of the order of $84.1,67.3$, and $60.2 \mathrm{~kJ} / \mathrm{mol}$ per ring. ${ }^{20}$ This means that the energy difference between phase $\mathrm{B}$ and phase A is $0.439,0.351$, and $0.314 \mathrm{eV}$ per ring. Since there are six bonds for a ring, $t_{e}$ will be $0.073,0.06$, and $0.052 \mathrm{eV}$ if it is assumed that the energy difference is well distributed in a ring and $t_{e}$ in a chain's situation is similar to $t_{e}$ in a ring's situation. So $t_{e}$ is evaluated as about $0.07-0.05$ $\mathrm{eV}$ for these conjugated polymers. From the viewpoint of $t_{e}$, negative polarization of the biexciton can be yielded in these conjugated polymers. Of course, the concrete value of $t_{e}$ depends on the real material, and the material with small $t_{e}$ is a preference in order to get negative polarization of the biexciton.

Dynamical studies ${ }^{21-23}$ show that the relaxation time of self-trapping excited states in low-dimensional systems is usually very short [about 70-200 fs (Refs. 21-23)]. Absorbing a photon can make a single-excited state become a biexcited state in conjugated polymers. The process inverts the polarization since the polarization direction of a singleexcited state is opposite to that of a biexcited state. Therefore, a new photoinduced phenomenon-photoinduced polarization could exist - photoinduced polarization inversion. The phenomenon might be used to make photoinduced ultrafast quantum switch devices or molecular switch devices,${ }^{24}$ since the relaxation time for the self-trapping excited states of electroluminescent polymers ${ }^{23}$ is quite short.

In addition, due to negative polarization, the electric capacity will decrease as the electric field increases. A possible result is negative permittivity, which could be observed in experiments one day, since this presentation of numerical analog calculations illustrates theoretically that negative polarization exists. Negative electrical conductance phenomena ${ }^{25}$ have been used as oscillators. A negative permittivity phenomenon could be discovered one day and would have its application. 
Furthermore, the discovery of a physically realizable system that has a negative polarization is very interesting, since it means an analogy exists between the magnetic properties of paramagnetism and diamagnetism and the electronic properties of normal and negative polarization. The existence of an electronic similarity to the magnetic properties of paramagnetism and diamagnetism could have potential practical applications, and such systems would exhibit novel electronic features. This might lead to a new technological direction, especially at the end of the silicon road. Therefore, the calculation results would be of interest both in basic sciences and in technology.

Our calculations have revealed that an electric field will transfer part of the charges in a self-trapping excited state of conjugated polymers. Thus the polarization or dissociation of a self-trapping excited state may occur according to the strength of electric fields. The behavior is different than that of an electron-hole pair in conventional inorganic semiconductors under the field. The difference stems from the fact that a conjugated polymer is a quasi-1D material while a conventional inorganic semiconductor is a three-dimensional material. The low-dimensional lattice has instability, e.g., for a ground state of a quasi-1D conjugated polymer, there are single and double bonds. The distance of two sites is spaced out in a single bond and is compressed in a double bond. So the lattice of a quasi-1D conjugated polymer is in a strained state, i.e., in a state of instability. When extra electron(s) and hole(s) exist, the strained lattice near the electron(s) and hole(s) will be relaxed. Thus the lattice is deformed, and the self-trapping excited states are induced. This is different than a 3D system. Since a 3D lattice is not strained, it will not be relaxed only because of extra electron(s) and hole(s). Only when the interactions of the electron lattice is very strong (e.g., ionic crystal) or some excited states (e.g., polaron) exist, will the extra electron(s) and hole(s) make their surrounding lattice distorted locally and form a polaron. However, a very weak interaction is enough to make a quasi-1D lattice relax, leading to some interesting phenomena [such as selftrapping excited states induced by extra electron(s) and hole(s), negative polarization, etc.] which are different than those in 3D systems. And these phenomena would be of great interest both fundamentally and technologically.

\section{SUMMARY}

By using numerical analog calculations, this presentation has revealed that the applied electric fields have strong effects on the self-trapping excited states of conjugated polymers. Weak or moderate fields will polarize the self-trapping excited states, and the degree of polarization increases linearly with the field $E$. The self-trapping single-excited state corresponds to normal polarization. It is worth mentioning that the self-trapping biexcited states of conjugated polymers correspond to negative polarization. This phenomenon is due to both the existence of two localized electronic states (the "'high level"' and the "low level') near the midgap in the self-trapping excited state and the opposite polarization direction of the two localized states. The latter can be understood according to the quantum theory of polarization. The significance of negative polarization and its possible uses are also discussed. Strong electric fields will split the selftrapping single-excited state directly into a hole polaron and an electron polaron, and the field annihilation of the selftrapping single-excited state (emitting species) leads to PL quenching. These calculations are in agreement with the relevant experiments. ${ }^{1-6}$

\section{ACKNOWLEDGMENTS}

One of the authors R.L.F., thanks Dr. Dai Ning for helpful discussions. This work was supported by the National Natural Science Foundation of China under Grant No. 19874068, and the National High Performance Computing Fund of China.
${ }^{1}$ W. Graupner, G. Cerullo, G. Lanzani, M. Nisoli, E. J. W. List, G. Leising, and S. De Silverstri, Phys. Rev. Lett. 81, 3259 (1998)

${ }^{2}$ S. Tasch, G. Kranzelbinder, G. Leising, and U. Scherf, Phys. Rev. B 55, 5079 (1997)

${ }^{3}$ R. Kersting, U. Lemmer, H.J. Bakker, R.F. Mahrt, H. Kurz, V.I. Arkhipov, H. Bässler, and E.O. Göbel, Phys. Rev. Lett. 73, 1440 (1994)

${ }^{4}$ V.I. Arkhipov, H. Bässler, M. Deussen, E.O. Göbel, R. Kersting, H. Kurz, U. Lemmer, and R.F. Mahrt, Phys. Rev. B 52, 4932 (1995)

${ }^{5}$ S. Tasch, A. Niko, G. Leising, and U. Scherf, Appl. Phys. Lett. 68, 1090 (1996).

${ }^{6}$ N. Pfeffer, D. Neher, M. Remmers, C. Poga, M. Hopmeier, and R. Mahrt, Chem. Phys. 227, 167 (1998).

${ }^{7}$ J.H. Burroughes, D.D.C. Bradley, A.R. Brown, R.N. Marks, K. Mackay, R.H. Friend, P.L. Burn, and A.B. Holmes, Nature (London) 347, 539 (1990).

${ }^{8}$ A.J. Heeger, S. Kivelson, J.R. Schrieffer, and W.P. Su, Rev. Mod. Phys. 60, 781 (1988).
${ }^{9}$ R.H. Friend, D.D. Bradley, and P.D. Townsend, J. Phys. D 20, 1367 (1987).

${ }^{10}$ X. Sun, J. Liu, C. Wu, and R. Fu, Prog. Phys. 10, 439 (1990).

${ }^{11}$ R. Fu, R. Fu, and X. Sun, Phys. Rev. B 48, 17615 (1993).

${ }^{12}$ R. Fu, Y. Hongjun, R. Fu, and X. Sun, Chin. J. Infrared Millim. Waves 12, 91 (1993).

${ }^{13}$ F. Rouli, Y. Hongjun, L. Lei, F. Rongtang, M. Jian, S. Xin, and Zhang Zhilin, Acta Phys. Sin. 47, 94 (1998).

${ }^{14}$ F. Rouli, J. Chu, R. Fu, L. Li, and X. Sun, J. Mater. Sci. Technol. 15, 354 (1999).

${ }^{15}$ F. Rouli C. Junhao, L. Lei, and S. Xin, Chin. J. Luminescence Suppl. 19, 139 (1998).

${ }^{16}$ S. Stafstrom and K.A. Chao, Phys. Rev. B 29, 7010 (1984).

${ }^{17}$ W.P. Su, J.R. Schrieffer, and A.J. Heeger, Phys. Rev. B 22, 2099 (1980).

${ }^{18}$ W.P. Su, Solid State Commun. 35, 899 (1980).

${ }^{19}$ F. Rouli, C. Junhao, J. Hao, and S. Xin, Chin. Phys. Lett. 16, 764 (1999).

${ }^{20}$ J.L. Bredas, B. Themans, J. Fripiat, and J. Andre, Phys. Rev. B 29, 6761 (1984). 
${ }^{21}$ F. Rouli, Y. Hongjun, F. Rongtang, Y. Zhigang, and S. Xin, Acta Phys. Sin. 43, 1336 (1994).

${ }^{22}$ R.-L. Fu, J.-H. Chu, G.-P. Zhang, Y.-S. Ma, X. Sun, K. Hag Lee, and T. Young Park, Commun. Theor. Phys. 26, 23 (1996)
${ }^{23}$ F. Rouli, Z. Erhai, and S. Xin, J. Infrared Millim. Waves 18, 184 (1999).

${ }^{24}$ F. Rouli and G. Guo, J. Infrared Millim. Waves, 19, 259 (2000).

${ }^{25}$ C. Kittel, Solid State Physics, 5th ed. (Wiley, New York, 1976). 